山્યFANÇAISE

$\supset \mathrm{DE}$

$\stackrel{1=1}{\simeq}$ PÉDAGOGIE

\section{Revue française de pédagogie}

Recherches en éducation

174 | janvier-mars 2011

Évaluation, développement professionnel et organisation scolaire

\title{
Vers un usage européen du modèle des communautés de pratique en formation des enseignants
}

Using models of communities of practice in teacher training at European level Hacia un uso europeo del modelo de las comunidades de práctica en formación de los docentes

Auf dem Weg zu einem europaweiten Gebrauch der Community of Practice in der Lehrerausbildung

\section{Guillaume Escalié et Sébastien Chaliès}

\section{(2) OpenEdition}

Journals

Édition électronique

URL : http://journals.openedition.org/rfp/2864

DOI : $10.4000 / \mathrm{rfp} .2864$

ISSN : 2105-2913

Éditeur

ENS Éditions

\section{Édition imprimée}

Date de publication : 15 mars 2011

Pagination : 107-118

ISBN : 978-2-7342-1203-4

ISSN : 0556-7807

Référence électronique

Guillaume Escalié et Sébastien Chaliès, «Vers un usage européen du modèle des communautés de pratique en formation des enseignants », Revue française de pédagogie [En ligne], 174 I janvier-mars 2011, mis en ligne le 15 mars 2015, consulté le 19 avril 2019. URL : http://journals.openedition.org/ rfp/2864; DOI : 10.4000/rfp.2864 


\section{Vers un usage européen du modèle des communautés de pratique en formation des enseignants Guillaume Escalié et Sébastien Chaliès}

Le renforcement du partenariat " université-écoles " constitue une des orientations adoptées dans le cadre des politiques éducatives européennes afin d'optimiser la formation des enseignants. Après l'avoir contextualisée institutionnellement, cet article montre en quoi et comment cette orientation invite à la mise en œuvre d'une nouvelle forme d'apprentissage professionnel, par le réaménagement des dispositifs usuels de formation, à partir du modèle des " communautés de pratique » (Lave \& Wenger, 1991). Sur la base d'une description des intérêts et des limites inhérents à ces nouveaux dispositifs, cet article questionne finalement la place que devraient occuper les établissements aux côtés de l'université dans le cadre de la formation des enseignants.

Descripteurs (TESE) : modèle de formation des enseignants, partenariat d'écoles, établissement de formation des enseignants.

Cet article propose une recension des principaux travaux nationaux et internationaux relatifs à la mise en œuvre de dispositifs de formation basés sur le modèle des "communautés de pratique " (Lave \& Wenger, 1991 ; Wenger, 1998) dans le cadre de la formation des enseignants. Elle est organisée en cinq parties. La première partie montre en quoi le renforcement du partenariat « université-écoles ${ }^{1}$ » constitue une des orientations adoptées dans le cadre des politiques éducatives européennes, notamment française, afin d'optimiser la formation des enseignants. La deuxième partie développe les fon- dements théoriques sous-jacents au modèle des communautés de pratique, exploité dans ce cadre pour parvenir à renforcer ce partenariat. La troisième partie détaille les intérêts et les limites de ce type d'exploitation. La partie suivante présente les principaux dispositifs mis en œuvre à partir du modèle des communautés de pratique. Pour conclure, la dernière partie montre enfin que, dans ce type de dispositif, les établissements, en permettant le rapprochement des situations de formation et de pratique professionnelle, occupent une place de plus en plus privilégiée dans le partenariat qui les lie à l'université. 


\section{UNE POLITIQUE EUROPÉENNE EN FAVEUR D'UN RENFORCEMENT DU PARTENARIAT UNIVERSITÉ-ÉCOLES}

Ces dernières années, l'accroissement de la qualité de la formation des enseignants est devenu une priorité au sein de l'Union européenne (Cros, 2005). L'amélioration de la formation des enseignants figure en effet parmi les axes prioritaires du programme Éducation et formation 2020, relatif aux objectifs futurs des systèmes d'éducation et de formation, entérinés par le Conseil de l'Union européenne à la suite du processus de Bologne (Conseil de l'Union européenne, 2009). En dépit des réformes introduites récemment dans différents pays européens, la formation initiale des enseignants, sous de nombreux aspects, ne les prépare pas efficacement à faire face à la complexité de leur métier (OCDE, 2009). Parmi les différentes composantes de cette formation, celle relative à la dimension professionnelle est largement discutée, voire dans certains pays repensée dans le cadre d'un nouvel examen des programmes de formation (Smith, 2008). Accompagnant ce mouvement de rénovation de la formation des enseignants, I'OCDE recommande ainsi par exemple que, dans le débat sur la durée de la formation des enseignants, les pays accordent plus d'importance à l'accroissement des ressources consacrées à l'intégration et au développement professionnel (OCDE, 2005). Plus concrètement, l'OCDE invite les pays européens à se donner les moyens d'optimiser les stages de pratique professionnelle menés dans les établissements et de renforcer les programmes d'insertion à la profession. Elle relève en ce sens par exemple que " les étapes de la formation initiale, de l'initiation et de la formation professionnelle des enseignants doivent être plus étroitement connectées pour élaborer un système d'apprentissage et de formation plus cohérent pour les enseignants » (OCDE, 2005, p. 11).

Dans le même ordre d'idées, la Commission des communautés européennes pointe, lors d'une communication intitulée "Améliorer la qualité des études et de la formation des enseignants 》 (Commission des communautés européennes, 2007), la faiblesse de la dimension professionnelle au cœur des formations des enseignants dans de nombreux États. Elle note ainsi par exemple que « dans de nombreux états membres, il n'existe guère de coordination systématique entre les différentes composantes de la formation des enseignants, ce qui entraîne un manque de cohérence et de continuité » (Commission des communautés européennes, 2007 , p. 3). En lien avec ce constat, cette commission fait des recommandations aux États afin qu'ils infléchissent leurs orientations politiques en matière de formation des enseignants (Commission européenne, 2005). Parmi ces recommandations, celle relative à la nécessité de penser les programmes de formation dans le cadre d'un véritable partenariat entre les universités et les établissements est tout particulièrement soulignée. Cette commission précise par exemple que "les établissements d'enseignement et de formation pour enseignants devraient placer l'organisation de leur travail sous le signe de la collaboration et du partenariat avec les écoles " (Commission des communautés européennes, 2007, p. 3). Elle rejoint là bon nombre d'auteurs qui vont jusqu'à avancer que la capacité des systèmes d'éducation et de formation à relever les défis $\mathrm{du} X \mathrm{XI}^{\mathrm{e}}$ siècle dépend des possibilités offertes aux enseignants par les politiques gouvernementales de bénéficier de " programmes de développement professionnel tout au long de la vie et basés sur la collaboration avec l'université » (Collinson, Kozina, Lin et al., 2009).

Politiquement introduite il y a une dizaine d'années, l'idée selon laquelle il est nécessaire de renforcer le partenariat université-écoles gagne donc du terrain dans les politiques européennes en matière de formation des enseignants. Plus spécifiquement, a été engagée en France une réforme de la formation initiale des enseignants s'inscrivant dans une volonté d'harmonisation des diplômes au niveau européen. La lettre de la Direction générale de l'enseignement supérieur du 17 octobre 2008 (MESR, 2008) introduisant cette réforme indique que l'un des principaux enjeux réside dans le maintien d'une formation professionnelle de qualité. Plus précisément, «la formation professionnelle ne saurait se limiter à l'envoi des étudiants dans les classes [...]. II est souhaitable qu'elle puisse s'articuler avec une réflexion didactique, pédagogique, disciplinaire et épistémologique qui suppose des allers-retours entre terrain et formation » (MESR, 2008, p. 2). Bien que l'organisation de la formation soit différente, le principe d'un partenariat entre l'université et les établissements d'accueil des enseignants en formation semble donc reconduit. En articulation avec la mise en place de masters ouvrant sur les différents métiers de l'enseignement, une nouvelle structuration de la formation professionnelle est par ailleurs envisagée en vue d'accompagner les enseignants dans leur entrée dans le métier (MEN, 2010). Ainsi l'année de prise de fonction devrait s'inscrire dans " un continuum de formation, en articulation avec celle qui leur a été dispensée antérieurement » (MEN, 2010, p. 1). 
L'articulation entre d'une part l'élévation du niveau de formation universitaire et d'autre part la multiplicité des vécus professionnels autorisée par la mise en place de stages de différente nature peut donc être associée à un enjeu majeur de la refonte de la formation des enseignants (Perrenoud, Altet, Lessard et al., 2008). Dans ce cadre, le réaménagement des dispositifs usuels de formation apparaît comme nécessaire afin de renforcer le lien entre les volets académique et professionnel de la formation.

\section{RENDRE EFFECTIF LE PARTENARIAT UNIVERSITÉ- ÉCOLES PAR LE MODĖLE DES COMMUNAUTÉS DE PRATIQUE}

Les orientations institutionnelles visant à renforcer le partenariat université-écoles trouvent un écho dans la littérature scientifique du domaine. En effet un grand nombre de travaux pointent la nécessité de redéfinir ce partenariat en faisant tomber les barrières traditionnellement érigées entre ces deux types d'établissements (Haymore Sandholtz, 2002 ; Mule, 2006). L'idée partagée par ces différents travaux est de promouvoir et mettre en œuvre une nouvelle forme d'apprentissage professionnel (Sim, 2006) en s'inspirant notamment du modèle des communautés de pratique (Lave \& Wenger, 1991), et plus particulièrement d'un de ses postulats fondateurs : participer à la communauté pour se former.

Dans ce modèle ancré sur une théorie sociale située de l'apprentissage, la formation professionnelle peut en effet être appréhendée comme la conséquence d'une participation à la communauté, sous la forme d'interactions sociales entre les différents acteurs (enseignants et formateurs) provenant de divers horizons institutionnels (Barcet, Bonamy, Esnault et al., 2004), et non comme le résultat d'un processus mécaniste planifié de transmission cognitive (Cox, 2005). La participation à la communauté est donc centrale dans l'apprentissage (Boylan, 2009) car elle permet la construction de connaissances assimilables à des significations négociées entre les membres de la communauté, c'est-à-dire à des expériences constitutives d'une pratique sociale partagée (Barcet, Bonamy, Esnault et al., 2004 ; Wenger \& Gervais, 2005). C'est en ce sens que Rogoff, Matusov et White (1996) précisent que « l'apprentissage et le développement se produisent lorsqu'une personne participe aux activités de sa communauté transformant ainsi sa compréhension, ses rôles et ses responsabilités au fil de sa participation "(Rogoff, Matusov \& White, 1996, p. 390).

En retour, cet apprentissage par la participation à la communauté de pratique modifie le mode de participation à cette communauté, permettant ainsi à l'apprenant de progressivement passer d'une participation périphérique de type « nouveau venu " (new comer) à une participation plus centrale de type " membre principal » (core member, voir Alexander, Van Wyck, Bereng et al., 2010 ; Charlier, 2010). En d'autres termes, l'apprentissage permet à l'apprenant de s'inscrire dans une participation à la communauté de plus en plus « légitime » (Lave \& Wenger, 1991), c'est-à-dire dans une participation davantage centrée sur le partage que sur l'échange et visant la construction de pratiques collectives et non plus seulement individuelles (Charlier, 2010). En outre, cette participation crée une histoire d'apprentissage qui change les rapports de l'apprenant à la pratique et aux autres membres engagés dans la communauté, et in fine transforme son identité. Cette dernière est en effet envisagée dans ce modèle comme le pivot entre les dimensions individuelle et sociale de l'apprentissage. La participation est alors le moyen par lequel l'identité de l'apprenant relative à la communauté de pratique peut évoluer (Barcet, Bonamy, Esnault et al., 2004) du statut de nouveau venu à celui de membre central. C'est en ce sens que certains auteurs insistent sur la "générativité 》 de l'identité des apprenants (Holland \& Lave, 2001), à travers l'évolution de la nature de leur participation à la communauté de pratique (Niesz, 2010). En envisageant l'apprentissage à travers le prisme de la participation, la théorie des communautés de pratique constitue donc un modèle qui imbrique l'individuel et le collectif. II ne s'agit pas ici de passer d'un modèle individuel de l'apprentissage à un modèle collectif, mais de considérer la construction de l'apprentissage dans une relation réflexive entre les pratiques sociales d'une communauté et les processus de construction individuelle (Mottier Lopez, 2008).

\section{ADOPTION DU MODÈLE DES COMMUNAUTÉS DE PRATIQUE EN FORMATION DES ENSEIGNANTS : INTÉRÊTS ET LIMITES}

Tel que le développe Wenger (1998) dans son texte portant sur le modèle des communautés de pratique, trois principaux intérêts peuvent y être associés du point de vue de la formation professionnelle. Le pre- 
mier est relatif à la nature de l'« engagement » des différents membres au sein de la communauté : les relations entre membres ne répondent pas à une logique prescriptive descendante, tel qu'il est traditionnellement possible de l'observer entre l'université et les établissements, au cœur desquels les conseillers pédagogiques ${ }^{2}$ (CP par la suite) et les enseignants novices (EN par la suite) ne parviennent que partiellement à saisir ce qui est attendu d'eux (Bullough, Draper, Smith et al., 2004). L'engagement des membres répond davantage en effet à des processus de négociation sur les significations à accorder aux expériences de métier abordées et débattues (Sim, 2006), rendant ainsi possibles l'apprentissage et le développement professionnel de chacun, qu'il soit apprenant ou formateur (Vescio, Ross \& Adams, 2008). Ce type d'aménagement permet plus précisément aux différents membres de la communauté, EN, CP et formateurs universitaires (FU dans la suite du texte), de s'inscrire progressivement dans un langage et des attentes professionnelles partagés (Nguyen, 2009). Ainsi par exemple, lorsqu'ils sont impliqués dans un dispositif de formation s'appuyant sur le modèle des communautés de pratique, les apprenants et leurs formateurs parviennent progressivement, lors de l'entretien de formation suivant la leçon, à se mettre d'accord sur la nature de l'objectif poursuivi lors de cette dernière ou encore sur la méthode à suivre pour la planifier avec davantage d'efficacité (Ottesen, 2007). Les échanges menés au cours de ces entretiens de formation peuvent donc être considérés comme des opportunités permettant aux acteurs de construire collectivement des accords sur les attentes de formation, relatives à la mise en œuvre de certaines compétences professionnelles en situation de classe (telle que " concevoir une leçon »). La construction de ces accords relatifs à des espaces de significations partagées est d'autant plus nécessaire qu'elle permet aux EN de passer d'une participation périphérique à une participation plus centrale au sein de la communauté, et ainsi de transformer de façon significative leurs pratiques professionnelles en classe (Moran, Abott \& Clark, 2009). Inversement, en l'absence de cette construction, les EN ont tendance à se perdre progressivement dans une " appréciation à plusieurs voies " de leur activité et in fine ne se développent pas professionnellement (Tilema, 2009).

Ce type d'engagement des différents membres de la communauté dans la construction négociée d'accords sur la signification des expériences de métier, abordées lors des entretiens de formation, modifie aussi les actions des formateurs et leur implication dans les plans de formation. II favorise ainsi par exemple pour les CP une meilleure explicitation du cahier des charges de la formation (Carver \& Katz, 2004) et des apports théoriques extraits de la recherche (Thies-Sprinthall, 1986), voire leur propre implication dans des recherches-actions (Twombly, Wolf-Wendel, Williams et al., 2006). Parallèlement, les FU trouvent dans ce type de partenariat avec les $\mathrm{CP}$ et les EN des « occasions de formation » (Murray, 2005). Ils parviennent ainsi par exemple à davantage interroger le métier d'enseignant dans ses dimensions génériques (Stotsky, 2006). Dans ce type de dispositifs de formation, les membres de la communauté constituent donc un groupe de professionnels qui collaborent, apprennent et tissent des liens interpersonnels (Horn \& Little, 2010), voire à terme qui se forgent une identité commune à partir d'un sentiment d'appartenance à la même communauté (Mello, 2003).

Nommé “ imagination ", le deuxième intérêt est relatif aux possibilités offertes par les dispositifs de formation, eux-mêmes pensés à partir du modèle des communautés de pratique, de mieux articuler en leur sein les composantes théorique et pratique de la formation, et par là-même d'atténuer le « choc de la pratique » pour les EN (Korthagen, 2010). Ce type de dispositif permet en effet de porter un regard nouveau sur les apports théoriques ordinairement délivrés à l'université, afin d'éviter qu'ils soient "effacés " (washed out) par les expériences vécues par les EN en situation de classe dans les établissements (Korthagen, 2010). C'est ici une réponse possible aux critiques adressées aux dispositifs de formation traditionnels, au sein desquels la délivrance des connaissances aux EN s'effectue pour une large partie à l'université, c'est-à-dire en dehors de tout contexte professionnel, et contribue ainsi très faiblement à la construction de leurs compétences professionnelles (Laksov, Mann \& Dahlgren, 2008). Porteurs de zones d'interactions critiques propices aux changements, les dispositifs de formation pensés à partir du modèle des communautés de pratique rendent au contraire possible l'expérimentation de nouveaux modèles pédagogiques autour d'“ objets frontières " (boundary objects) tels que l'enseignement, l'apprentissage ou les savoirs (Gorodetsky \& Barak, 2008), positionnés entre apports théoriques et pratique effective de classe. Pour chacun de ces "objets ", les formateurs et les EN cherchent en effet collectivement à associer aux définitions théoriques délivrées à l'université divers usages possibles en contexte professionnel. Ils construisent en ce sens progressivement 
la " cohérence du curriculum de formation " (Lamb \& Jacobs, 2009). Au final, ce travail collectif aboutit à l'acquisition par les EN d'une « théorie pratique " (Buitink, 2009), constituée de connaissances et compétences professionnelles toujours situées et négociées entre membres de la communauté, et par làmême à la transformation effective de leur pratique professionnelle (Gorodetsky \& Barak, 2008).

Nommé « alignement », le dernier intérêt associé aux dispositifs de formation pensés à partir du modèle des communautés de pratique est relatif à l'atténuation d'une répartition formelle (et le plus souvent exclusivement administrative) des responsabilités entre les différents membres, au profit d'une collaboration plus effective au quotidien dans les établissements (Melville \& Bartley, 2009). Sutherland, Scanlon et Sperring (2005) ont par exemple montré que des EN ayant eu l'occasion de recevoir une formation dans ce type de dispositif tirent profit de cette collaboration pour modifier plus facilement leur pratique professionnelle que ceux impliqués dans un système usuel de formation. En atténuant les rapports hiérarchiques usuellement établis entre l'université et les établissements, la mise en place de ce type de dispositif de formation permet en effet de créer des " opportunités d'apprentissage élargies 》 (Zeichner, 2010), et donc la construction et le développement de nombreuses compétences professionnelles chez les EN. Parmi ces compétences, celle relative à l'adaptation de leur enseignement à une plus grande diversité d'élèves (et notamment aux élèves handicapés, voir Mortier, Hunt, Leroy et al., 2010) est tout particulièrement alimentée par la mise en place de dispositifs de formation favorisant un " enseignement collaboratif " (Nevin, Thousand \& Villa, 2009). Formés dans ce type de dispositif, les EN parviennent par ailleurs à instaurer plus rapidement une ambiance de classe propice au travail, favorisant des progrès plus marqués chez tous les élèves (Stanulis \& Folden, 2009). En retour, ils développent de façon significative une confiance en eux et un sentiment d'auto-efficacité (Helfeldt, Capraro, Capraro et al., 2009).

Certains travaux restent toutefois plus critiques quant aux bénéfices liés à la mise en place de dispositifs de formation pensés à partir du modèle des communautés de pratique. Ces travaux sont à mettre en perspective avec la récente évolution de ce modèle sous l'influence d'une idéologie managériale (Watson, 2002). L'analyse de travaux récents (voir par exemple Wenger, McDermott \& Snyder, 2002) laisse en effet transparaître une redéfinition fondamentale du concept de «communauté de pratique » et son utilisation comme outil de gestion des connaissances (Cox, 2005). Comme le précise cet auteur, ce concept servait initialement d'outil pour apprécier la construction (ou non) de significations partagées entre les acteurs en situation d'interaction sociale. Actuellement il est de plus en plus utilisé pour optimiser la gestion de groupes communautaires ou informels, patronnés par une organisation pour faciliter le partage de connaissance ou l'apprentissage.

Dans cette perspective, un des points de discussion de la littérature porte sur la gestion pédagogique, administrative et financière des dispositifs de formation pensés à partir du modèle des communautés de pratique (Castle, Fox \& O'Hanlan Souder, 2006). La volonté initialement affichée d'une prise de décisions conjointe et partagée entre les différents membres de la communauté est, en effet, régulièrement contrariée par les résistances de l'université et des établissements à partager les responsabilités qui leur sont traditionnellement attribuées (Fullan, Galluzzo, Morris et al., 1998). Outre les difficultés relatives à la mise en œuvre d'une gouvernance partagée, certaines études notent aussi la forte prégnance des contingences locales économiques (Rice, 2002), politiques (Yendol-Silva \& Fichtman Dana, 2004) et/ou sociales (Teitel, 1998) sur l'efficacité de tels dispositifs. Cette difficulté est d'autant plus marquée que l'université et les établissements sont assimilables à des systèmes d'activités dont les attentes et les finalités divergent (Smagorinsky, Cook, Jackson et al., 2004). Tendre à une responsabilité partagée de la formation aboutit ainsi souvent à une forme de " collusion » entre rôles et responsabilités de chacun (Cartaut \& Bertone, 2009), source de déstabilisation chez les EN (Bullough, Draper, Smith et al., 2004). Ces derniers finissent ainsi par entretenir un rapport quasi exclusif de formation soit avec les CP (Larose, Lenoir, Grenon et al., 2000), soit avec les FU (Smith \& Lev-Ari, 2005). Un parallèle peut semblet-il être ici tracé avec la distinction posée par Charlier (2010) entre les activités d'échange et de partage entre les membres de la communauté. Selon cet auteur, l'intérêt du modèle des communautés de pratique ne réside pas seulement dans la gestion managériale des différents membres et de leur activité d'échange de pratiques. Penser ces dispositifs de formation permet de s'interroger sur leur activité de partage, c'est-à-dire leur activité pour « prendre part » à une discussion autour d'un objet, d'une idée, d'une pratique, placés au service des différents membres de la communauté. 


\section{EXEMPLES DE DISPOSITIFS DE FORMATION MIS EN CEUVRE À PARTIR DU MODĖLE DES COMMUNAUTÉS DE PRATIQUE}

Un grand nombre de publications rend compte des modalités de mise en œuvre de divers dispositifs de formation des EN pensés sur le modèle des communautés de pratique (voir par exemple Haymore Sandholtz, 2002 ; Chaliès, Cartaut, Escalié et al., 2009 ; Whitehead \& Fitzgerald, 2007 ; Zeichner, 2006). Parmi ces dispositifs, celui dit des Professional Development Schools est largement diffusé et étudié. II a été initialement mis en œuvre aux États-Unis à partir du second rapport du Holmes Group (1990), préconisant une restructuration de la formation des enseignants afin de promouvoir cette profession, de garantir l'application des réformes éducatives et de proposer un enseignement adapté à tous les publics scolaires (Darling-Hammond, 1994 ; Zeichner, 1992). Pour y parvenir, des collaborations entre plusieurs établissements d'un même ou de différents districts scolaires et une ou plusieurs universités ont été pensées afin d'aboutir à la mise en œuvre de nouvelles " organisations apprenantes" (Ross, 1995). Au sein de ces organisations, les FU, les CP et les EN cherchent à travailler en étroite collaboration. Ainsi par exemple, tous les enseignements théoriques sont réalisés conjointement par les $\mathrm{FU}$ et les $\mathrm{CP}$ à l'établissement et/ou à l'université et pensés dans une perspective d'exploitation immédiate par les EN en situation de pratique professionnelle (DarlingHammond, 1994). Dans le même ordre d'idées, chaque activité de supervision et/ou de conseil à partir des cours donnés par les EN est conduite communément par les CP et les FU impliqués de manière quasi quotidienne dans les établissements (Grisham, Bergeron, Brink et al., 1999).

Ce type de dispositif a aussi été introduit dans certains pays européens. Ainsi, en Finlande par exemple, les efforts menés au sein des masters consacrés à la formation des enseignants afin de rapprocher les enseignements théoriques et la pratique de classe ont abouti à l'institution de partenariats locaux (Jakku-Sihvonen \& Niemi, 2006) entre la faculté de formation des enseignants de l'université et le réseau adjacent d'établissements appelés "écoles normales» (Meisalo, 2007). Au sein de ce réseau, les $\mathrm{CP}$ jouent un double rôle d'enseignant et de conseiller. Leur collaboration avec les FU est nourrie par leur implication dans des travaux de recherche relatifs aux problématiques d'apprentissage et d'enseignement rencontrées au quotidien dans les établissements par les EN. Dans le même ordre d'idées, des études menées en Amérique du Nord montrent que, dans ce type de dispositifs établis entre une université et un ou plusieurs établissements « associés " (Raymond \& Lenoir, 1998), le clivage entre les fonctions des FU et des CP disparaît progressivement (Burton, 1998) pour laisser place à un travail collectif mené par la " communauté enseignante élargie » (Wang, Strong \& Odell, 2004). Parmi ce type de dispositif, celui des " centres de pédagogie » (Goodlad, 1990), expérimenté aux États-Unis, occupe une place particulière. Vis-à-vis de l'université, ces centres sont en effet pensés et organisés comme des structures autonomes sur les plans budgétaire et pédagogique. Structurellement proches de ce qu'étaient les instituts universitaires de formation des maîtres (IUFM) en France avant la mise en place de la mastérisation de la formation des maîtres, ces centres ne fonctionnent pas toutefois de façon isolée. Ils constituent un trait d'union entre l'université et les établissements environnants, qui accueillent pour leur formation les étudiants ou les EN. Ces centres, comme les nouveaux IUFM devenus des écoles internes d'université, ont pour projet de concourir à la "professionnalisation de l'offre de formation et des enseignants ${ }^{3} »$.

Si la structuration des dispositifs peut varier, ces derniers tendent tous, en prenant appui sur le modèle des communautés de pratique, à une modification des modalités de travail entre les différents membres impliqués dans la formation. Ainsi par exemple, le dispositif dit des "groupes d'amis critiques » (Kwan \& Lopez-Real, 2005) est pensé pour permettre à des collectifs de formation constitués d'EN, de CP et de FU de mettre en œuvre et de mener des séquences d'analyse de pratique professionnelle à partir des verbalisations faites par les EN de leur activité d'enseignement. La construction de ce type de " collectif de formation » (Cartaut \& Bertone, 2009) n'est toutefois pas sans poser des difficultés, comme celle relative à la capacité des formateurs à s'entendre pour organiser les conditions et modalités d'une position complémentaire fondée sur leurs compétences singulières et leurs disponibilités (Awaya, McEwan, Heyler et al., 2003 ; Perry, Hutchinson \& Thauberger, 2008), afin de répondre au mieux aux besoins identifiés en amont avec les EN (Tsui, Lopez-Real, Law et al., 2001). Cette difficulté est d'ailleurs d'autant plus prégnante que certains travaux récents invitent à intégrer un nouveau membre au sein de cette communauté, pour aider à la constitution de cette complémentarité. On peut ainsi relever certaines 
initiatives locales comme par exemple la mise en place d'“ enseignants référents " à l'échelle d'un établissement ou d'un réseau d'établissements (Velzen \& Volman, 2009). Autrement nommés « enseignants éducateurs » (Velzen, Bezzina \& Lorist, 2009) ou encore « coordonnateurs de communauté " (Zeegers, 2005), ils sont toujours à la fois enseignants et formateurs et ont en plus une fonction de médiation (He, 2009) auprès des EN, CP et FU, pour faciliter l'engagement de tous dans la communauté de pratique (Escalié \& Chaliès, 2009).

\section{LES ÉTABLISSEMENTS AU CCEUR DES PROBLÉMATIQUES D'ÉDUCATION ET DE FORMATION}

Une des orientations actuelles de la politique européenne en termes de formation professionnelle des enseignants consiste à renforcer le partenariat université-écoles. Parmi les éléments de réponse fournis par la littérature scientifique du domaine, la mise en œuvre de nouveaux dispositifs de formation prenant appui sur le modèle des communautés de pratique occupe une place de choix. Même si elle a déjà fait l'objet de travaux, cette mise en œuvre pose encore toutefois un certain nombre de questions. Dans ce type de dispositifs, l'articulation entre les situations de formation à l'université et/ou dans les établissements et les situations de pratique professionnelle nécessite en effet d'être repensée dans une nouvelle dynamique dite d'" alternance intégrative » (Bucheton, 2009). Plus exactement, l'assimilation des expériences pratiques de classe comme à la fois objet, mais aussi support de formation et de professionnalisation (Mayen, 2007), bouscule la « pluralité de l'alternance » (Vanhulle, Merhan \& Ronveau, 2007) jusqu'ici mise en œuvre et pose un certain nombre de questions. En plaçant l'expérience pratique de classe au cœur du processus de formation (Moussay, Étienne \& Méard, 2009), ces dispositifs déplacent en effet le centre de gravité de la formation (Zeichner, 2006) et modifient à la fois l'" alternance institutionnelle » établie entre l'université et les établissements, l'« alternance organisationnelle » entre les situations de formation et la pratique de classe, mais aussi l'« alternance actorielle » en invitant à repenser les rapports entre l'individuel et le collectif dans le travail (Vanhulle, Merhan \& Ronveau, 2007).

Malgré « une certaine continuité dans le changement » (Brisard \& Malet, 2004) en termes de concep- tion de l'enseignement et de la formation, certaines études ont toutefois été déjà engagées sur les conséquences de la mise en œuvre de dispositifs au sein desquels les établissements sont pensés et gérés comme des " communautés d'apprentissage professionnel » (Keay, 2009). Assimilés à des « établissements apprenants » (Bouvier, 2001 ; Gather Thurler, 2000), à des "établissements scolaires formateurs" (Étienne, 1999) ou encore plus récemment à des “ organisations apprenantes » (Paquay, 2005 ; Krecic \& Grmek, 2008), ces établissements contribuent de façon plus prononcée à la formation, notamment en permettant la construction d'un « professionnalisme collectif [prenant le pas sur] l'individualisme " (Lessard, 2005a). Assimilables à des "espaces hybrides » (Zeichner, 2010) au cœur desquels est rendue possible une articulation effective et efficace du travail des différents membres de la communauté (Smith \& Evans, 2008), ces établissements sont une source de développement professionnel pour chacun (Coffey, 2010). Borko, Elliott et Uchiyama (2002) montrent ainsi par exemple que, dans ce type d'établissements, les EN construisent des connaissances, compétences et dispositions à enseigner, tout en œuvrant à la construction d'une communauté professionnelle partagée avec les autres membres apprenants ou formateurs, ce qui au final aboutit à un renforcement de la cohérence d'ensemble des enseignements dispensés aux élèves.

Les intérêts de la mise en œuvre de dispositifs de formation pensés à partir du modèle des communautés de pratique dépassent en outre le cadre de la formation initiale des enseignants. Certains travaux montrent, en effet, l'impact de tels dispositifs sur leur formation continue. Des résultats de recherche récents invitent d'ailleurs à repenser les partenariats entre l'université et les établissements pour permettre, au sein de ces derniers, la poursuite de l'accompagnement professionnel des enseignants après leur formation initiale (Mitchell, Reilly \& Logue, 2009). D'autres études montrent que ce type de dispositifs de formation présente aussi un intérêt dans le cadre de la formation de formateurs. Au sein de ces dispositifs, les établissements deviennent en effet un véritable lieu de formation, notamment pour les CP, grâce par exemple à la mise en place de communautés de conseil réunissant des $\mathrm{CP}$ et des $\mathrm{FU}$, mais aussi d'autres partenaires de la communauté éducative tels que les chefs d'établissement (Bryan \& Carpenter, 2008). Fondé sur la mise en synergie de formateurs au cœur des établissements (Crasborn, Hennissen, Brouwer et al., 2008), ce nouvel environnement de formation aiderait les CP à s'engager dans une relec- 
ture générique du métier d'enseignant, essentielle pour éviter l'écueil d'un accompagnement des EN exclusivement fondé sur leurs styles singuliers d'enseignement (Parker-Katz \& Bay, 2008).

Cependant le nombre encore limité de ce type d'études montre combien "les discours généraux des réformes et politiques éducatives sont encore à l'épreuve des réalités diverses du monde enseignant, du fonctionnement des établissements et de l'évolution de la formation des maîtres " (Lessard, 2005 b, p. 14). C'est donc sans nul doute ici l'un des principaux chantiers à entreprendre en termes de recherches en sciences de l'éducation et de la formation, afin d'accompagner au mieux ce " tournant organisationnel » (Malet \& Brisard, 2005) au sein du système éducatif français.

Guillaume Escalié DATIEF, université de Nice-Sophia Antipolis

Sébastien Chaliès sebastien.chalies@toulouse.iufm.fr UMR EFTS, IUFM Midi-Pyrénées, université Toulouse 2-Le Mirail

\section{NOTES}

1 Nous traduisons ici la terminologie la plus fréquemment exploitée (university-school) au cœur de la littérature scientifique. Par convention, nous entendons par « université-écoles » le partenariat établi entre l'université et un ou plusieurs établissements scolaires de premier ou second degré.

2 Les conseillers pédagogiques sont des enseignants en poste qui ont en charge une ou plusieurs classes, tout en étant impliqués comme formateurs dans le programme de formation professionnelle initiale des EN. Selon les pays, ils sont nommés associate teachers, mentor, mentor teacher, school-based mentor, cooperating teacher, induction tutor, induction supporter, classroom teacher ou encore school-based teacher educator (pour une synthèse, voir Hennissen, Crasborn, Brouwer et al., 2008). Les formateurs universitaires (FU dans le texte) sont généralement chargés d'enseignement et/ou de recherche dans les universités ou dans les centres de formation selon les pays et interviennent dans les programmes de formation professionnelle initiale et continue des enseignants. Certains ont une expérience d'enseignant et/ ou de conseiller pédagogique. Selon les pays, ils sont nommés university supervisor, student teachers supervisor, teacher training tutor, university tutor ou encore university-based teacher educator (Pour plus de détails, voir Chaliès, Cartaut, Escalié et al., 2009).

3 Nous faisons ici référence au colloque organisé par la conférence des directeurs d'IUFM ayant eu lieu à Paris en 2010: “20 ans de formation et de recherche dans les IUFM, et maintenant?

\section{BIBLIOGRAPHIE}

ALEXANDER G., VAN WYK M., BERENG T. \& NOVEMBER I. (2010). « Legitimate peripheral participation (LPP): The case for recognition of prior learning sites and knowledge in South Africa's transforming education system ". Teaching and Teacher Education, vol. 26, $\mathrm{n}^{\circ} 1$, p. $45-52$.

AWAYA A., McEWAN H., HEYLER D. et al. (2003). "Mentoring as a journey ". Teaching and Teacher Education, vol. 19, $\mathrm{n}^{\circ}$ 1, p. 45-56.

BARCET A., BONAMY J., ESNAULT L. et al. (2004). "Communautés de pratiques, management de la connaissance et production de service". Communication présentée à la conférence RESER, Castres. Disponible sur Internet à l'adresse : <http://fr. reser.net/Actes-du-congres-RESER-2004_a331.html> (consulté le 26 janvier 2011).

BORKO H., ELLIOTT R. \& UCHIYAMA K. (2002). "Professional development: A key to Kentucky's educational reform effort ". Teaching and Teacher Education, vol. 18, $\mathrm{n}^{\circ}$ 8, p. 969-987.

BOUVIER A. (2001). L'établissement scolaire apprenant. Paris : Hachette.
BOYLAN M. (2009). "Ecologies of participation in school classrooms ». Teaching and Teacher Education, vol. 25, $\mathrm{n}^{\circ} 1$, p. 1-10.

BRISARD E. \& MALET R. (2004). "Évolution du professionnalisme enseignant et contextes culturels ". Recherche et formation, $\mathrm{n}^{\circ} 45, \mathrm{p} .131-149$.

BRYAN H. \& CARPENTER C. (2008). " Mentoring: A practice developed in community? "Profesional Development in Education, vol. 34, n 1, p. 47-59.

BUCHETON D. (2009). L'agir enseignant : des gestes professionnels ajustés. Toulouse : Octarès.

BUITINK J. (2009). "What and how do student teachers learn during school-based teacher education ". Teaching and Teacher Education, vol. 25, $\mathrm{n}^{\circ}$ 1, p. 118127.

BULLOUGH R., DRAPER R., SMITH L. \& BIRRELL J. (2004). "Moving beyond collusion: Clinical faculty and university/public school partnership ». Teaching and Teacher Education, vol. 20, n 5, p. 505-521.

BURTON D. (1998). " The changing role of the university tutor within school-based initial teacher education: Issues of role contingency and complementarities within a secondary partnership scheme $»$. Journal of Education for Teaching, vol. 24, n² 2, p. 129-146. 
CARTAUT S. \& BERTONE S. (2009). « Co-analysis of work in the triadic supervision of preservice teachers based on neo-Vygotskian activity theory: Case study from a French university institute of teacher training ". Teaching and Teacher Education, vol. 25, n 8, p. 1086-1094.

CARVER C. \& KATZ D. (2004). « Teaching at the boundary of acceptable practice. What is new teacher mentor to do? " Journal of Teacher Education, vol. 55, $\mathrm{n}^{\circ} 5$, p. 449-462.

CASTLE S., FOX R. \& O'HANLAN SOUDER K. (2006). « Do Professional Development Schools (PDSs) make a difference? A comparative study of PDS and non-PDS teacher candidates ". Journal of Teacher Education, vol. $57, n^{\circ} 1$, p. $65-80$.

CHALIÈS S., CARTAUT S., ESCALIÉ G. \& DURAND M. (2009). «D'une utilité discutée à une utilité éprouvée du tutorat en formation initiale des enseignants : la nécessité d'une formation des tuteurs ". Recherche et formation, $n^{\circ} 61$, p. 85-129.

CHARLIER B. (2010). « L'échange et le partage de pratiques d'enseignement au cœur du développement professionnel ». Education \& formation, $n^{\circ} 293$, p. 137 149.

COFFEY H. (2010). " "They taught me": The benefits of early community-based field experiences in teacher education ". Teaching and Teacher Education, vol. 26, $\mathrm{n}^{\circ} 2$, p. 335-342.

COLLINSON V., KOZINA E., LIN Y. et al. (2009). "Professional development for teachers: A world of change ". European Journal of Teacher Education, vol. 32, n ${ }^{\circ} 1$, p. 3-19.

COMMISSION DES COMMUNAUTÉS EUROPÉENNES (2007). "Améliorer la qualité des études et de la formation des enseignants ". Communication de la Commission au Conseil et au Parlement européen. Bruxelles : Commission des communautés européennes. Disponible sur Internet à l'adresse : <http://europa.eu/legislation_summaries/education_ training_youth/lifelong_learning/c11101_fr.htm > (consulté le 26 janvier 2011).

COMMISSION EUROPÉENNE (2005). « Projet de principes européens communs concernant les compétences et les qualifications des enseignants ". Bruxelles : Commission européenne. Disponible sur Internet à l'adresse : <http://etuce.homestead.com/ publications2005/principles_fr.pdf> (consulté le 26 janvier 2011).

CONSEIL DE L'UNION EUROPÉENNE (2009). «Conclusions du Conseil du 12 mai 2009 concernant un cadre stratégique pour la coopération européenne dans le domaine de l'éducation et de la formation ("Éducation et formation 2020") ". Journal officiel de l'Union européenne, 28 mai 2009, C 119. Disponible sur Internet à l'adresse : <http://eur-lex.europa.eu/ LexUriServ/LexUriServ.do?uri=CELEX:52009XG0528\% 2801\%29:FR:NOT> (consulté le 26 janvier 2011).

COX A. (2005). "What are communities of practice? A comparative review of four seminal works ». Journal of Information Science, vol. 31, $\mathrm{n}^{\circ} 6$, p. 527-540.

CRASBORN F., HENNISSEN P., BROUWER N. et al. (2008). " Promoting versatility in mentor teachers' use of supervisory skills ». Teaching and Teacher Education, vol. $24, n^{\circ} 3$, p. $499-514$.
CROS F. (2005). Préparer les enseignants à la formation tout au long de la vie, une priorité européenne? Paris : L'Harmattan.

DARLING-HAMMOND L. (1994). An essay-review of Professional Development Schools: School for developing a profession. New York : Teachers College Press.

ESCALIÉ G. \& CHALIÈS S. (2009). « Optimiser le conseil pédagogique: la mise au travail d'un collectif de formation ". Travail et formation en éducation, $\mathrm{n}^{\circ} 4$

ÉTIENNE R. (1999). “ L'établissement formateur. Quelle contribution du "terrain" à la formation générale des professionnels de l'enseignement secondaire ? ” Recherche et formation, $\mathrm{n}^{\circ}$ 31, p. 137-151.

FULLAN M., GALLUZZO G., MORRIS P. \& WATSON N. (1998). The rise and stall of teacher education reform. Washington : American Association of Colleges for Teacher Education.

GATHER THURLER M. (2000). Innover au cœur de l'établissement scolaire. Paris : ESF.

GOODLAD J. (1990). Teachers for our nation's schools. San Francisco : Jossey-Bass.

GORODETSKY M. \& BARAK J. (2008). « The educationalcultural edge: A participative learning environment for co-emergence of personal and institutional growth ". Teacher and Teaching Education, vol. 25, n 7 , p. 19071918.

GRISHAM D., BERGERON B., BRINK B. et al. (1999). " Connecting communities of practice through Professional Development School activities ». Journal of Teacher Education, vol. 50, n³, p. 182-191.

HAYMORE SANDHOLTZ J. (2002). « Inservice training or professional development: Contrasting opportunities in a school/university partnership ». Teaching and Teacher Education, vol. 18, $\mathrm{n}^{\circ} 7$, p. 815-830.

HE A. (2009). « Bridging the gap between teacher educator and teacher in a community of practice: A case of brokering ». System, vol. 37, $\mathrm{n}^{\circ} 1, \mathrm{p} .153-163$.

HELFELDT J., CAPRARO R., CAPRARO M., FOSTER E. \& CARTER N. (2009). «An urban schools-university partnership that prepares and retains quality teachers for "high need" schools ". The Teacher Educator, vol. $44, n^{\circ} 1$, p. 1-20.

HENNISSEN P., CRASBORN F., BROUWER N., KORTHAGEN F. \& BERGEN T. (2008). « Mapping mentor teacher's roles in mentoring dialogues ». Educational Research Review, vol. 3, $\mathrm{n}^{\circ} 2$, p. 168-186.

HOLLAND D. \& LAVE J. (2001). « History in person: An introduction ». In D. Holland \& J. Lave (dir.), History in person: Enduring struggles, contentious practice, intimate identities. Santa Fe : School of American Research Press, p. 3-33.

HOLMES GROUP (1990). Tomorrow's schools: Principles for the design of Professional Development Schools. East Lansing : Holmes Group.

HORN I. \& LITTLE J. (2010). «Attending to problems of practice: Routines and resources for professional learning in teachers' workplace interactions ». American Educational Research Journal, vol. 47, $\mathrm{n}^{\circ} 1$, p. 181-217. 
JAKKU-SIHVONEN R. \& NIEMI H. (2006). « Introduction to the Finnish education system and teachers' work ". In R. Jakku-sihvonen \& H. Niemi (dir.), Research-based teacher education in Finland: Reflections by Finnish teacher educators. Jyväskylä : Finnish Educational Research Association, p. 7-13.

KEAY J. (2009). " Being influenced or being an influence: New teachers' induction experiences ". European Physical Education Review, vol. 15, n² 2, p. 225-242.

KORTHAGEN F. (2010). "Situated learning theory and the pedagogy of teacher education: Towards an integrative view of teacher behavior and teacher learning ". Teacher and Teaching Education, vol. 26, $\mathrm{n}^{\circ} 1$, p. 98-106.

KRECIC M. \& GRMEK M. (2008). «Cooperative learning and team culture in schools: Conditions for teachers' professional development ". Teaching and Teacher Education, vol. $24, \mathrm{n}^{\circ} 1$, p. 59-68.

KWAN T. \& LOPEZ-REAL F. (2005). “ Mentors' perceptions of their roles in mentoring student teachers ». AsiaPacific Journal of teacher education, vol. 33, $\mathrm{n}^{\circ} 3$, p. 275-287.

LAKSOV K., MANN S. \& DAHLGREN L. (2008). « Developing a community of practice around teaching: A case study ". Higher Education Research and Development, vol. 27, $\mathrm{n}^{\circ} 2$, p. 121-132.

LAMB L. \& JACOBS V. (2009). « Establishing and maintaining program coherence in a cohort-based graduate program ». The Teacher Educator, vol. 44, n² 2, p. 126142.

LAROSE F., LENOIR Y., GRENON V. \& SPALLANZANI C. (2000). "Les représentations des futurs enseignants québécois du primaire au regard de la formation initiale et des responsabilités des formateurs ". European Journal of Teacher Education, vol. 23, $n^{\circ} 3$, p. 275-288.

LAVE J. \& WENGER E. (1991). Situated learning: Legitimate peripheral participation. Cambridge : Cambridge University Press.

LESSARD C. (2005a). « Collaboration au travail : norme professionnelle et développement d'une pratique d'enseignement $"$. In D. Biron, M. Cividini \& J.-F. Desbiens, La profession enseignante au temps des réformes. Sherbrooke : Éd. du CRP, p. 435-458.

LESSARD C. (2005b). "Travailler ensemble? Des réformes éducatives aux pratiques enseignantes ». Recherche et formation, $\mathrm{n}^{\circ} 49$, p. 5-15.

MALET R. \& BRISARD E. (2005). « Travailler ensemble dans l'enseignement secondaire en France et en Angleterre ". Recherche et formation, $n^{\circ} 49$, p. 17-33.

MAYEN P. (2007). « Passer du principe d'alternance à l'usage de l'expérience en situation de travail comme moyen de formation et de professionnalisation ". In F. Merhan, C. Ronveau \& S. Vanhulle (dir.), Alternance en formation. Bruxelles: De Boeck, p. 83-100.

MEISALO V. (2007). « Subject teacher education in Finland: A research-based approach ». In R. Jakku-Sihvonen \& H. Niemi (dir.), Education as societal contributor. Bruxelles : Peter Lang, p. 161-180.

MELLO R. (2003). "The integrated cohort program: An evaluation of a pre-professional course of study ». The Educational Forum, vol. 67, n 4, p. 354-363.
MELVILLE W. \& BARTLEY A. (2009). " Mentoring and community: Inquiry as stance and science as inquiry ". International Journal of Science Education, vol. 32, $\mathrm{n}^{\circ} 6$, p. 807-828.

MINISTÈRE DE L'ÉDUCATION NATIONALE (2010). « Dispositif d'accueil, d'accompagnement et de formation des enseignants stagiaires des premier et second degrés et des personnels d'éducation stagiaires ». Circulaire n²010-037 du 25 février 2010. Bulletin officiel de l'Éducation nationale, 1 er avril 2010, $\mathrm{n}^{\circ} 13$.

MINISTÈRE DE L'ENSEIGNEMENT SUPÉRIEUR ET DE LA RECHERCHE (2008). " Campagne d'habilitation de diplômes de master pour les étudiants se destinant aux métiers de l'enseignement. Rentrée universitaire 2009 ". Lettre du 17 octobre 2008 à Mesdames et messieurs les présidents d'université et directeurs d'établissement d'enseignement supérieur. Paris : Ministère de l'Enseignement supérieur et de la Recherche. Disponible sur Internet à l'adresse : <http://www. etatsgeneraux-formationdesenseignants.fr/IMG/ pdf/2008-10-17a.pdf> (consulté le 26 janvier 2011).

MITCHELL S., REILLY R. \& LOGUE M. (2009). « Benefits of collaborative action research for the beginning teacher ". Teaching and Teacher Education, vol. 25, $\mathrm{n}^{\circ} 2$, p. 344-349.

MORAN A., ABOTT L. \& CLARK L. (2009). "Re-conceptualizing partnerships across the teacher education continuum ". Teaching and Teacher Education, vol. 25, $\mathrm{n}^{\circ}$ 7, p. 951-958.

MORTIER K., HUNT P., LEROY M., VAN DE PUTTE I. \& VAN HOVE G. (2010). "Communities of practice in inclusive education ». Educational Studies, vol. $36, \mathrm{n}^{\circ} 3$, p. 345-355.

MOTTIER LOPEZ L. (2008). Apprentissage situé : la microculture de classe en mathématiques. Berne : Peter Lang.

MOUSSAY S., ÉTIENNE R. \& MÉARD J. (2009). « Le tutorat en formation initiale des enseignants : orientations récentes et perspectives méthodologiques ". Revue française de pédagogie, $\mathrm{n}^{\circ} 166$, p. 59-69.

MULE L. (2006). “Preservice teachers' inquiry in a Professional Development School context: Implications in the practicum ". Teacher and Teaching Education, vol. $22, n^{\circ} 2$, p. $205-218$.

MURRAY J. (2005). "Re-addressing the priorities: New teacher educators and induction into higher education ». European Journal of Teacher Education, vol. $28, n^{\circ} 1$, p. 67-85.

NEVIN A., THOUSAND J. \& VILLA R. (2009). « Collaborative teaching for teacher educators: What does the research say? " Teaching and Teacher Education, vol. 25, $\mathrm{n}^{\circ} 4$, p. 569-574.

NGUYEN H. (2009). «An inquiry-based practicum model: What knowledge, practices, and relationships typify empowering teaching and learning experiences for student teachers, cooperating teachers and college supervisors? "Teaching and Teacher Education, vol. 25, $\mathrm{n}^{\circ}$ 6, p. 655-662.

NIESZ T. (2010). "Chasms and bridges: Generativity in the space between educators' community of practice ". Teaching and Teacher Education, vol. 26, $\mathrm{n}^{\circ} 1$, p. 37-44. 
OCDE (2005). Le rôle crucial des enseignants. Attirer, former et retenir des enseignants de qualité. Paris : OCDE.

OCDE (2009). Creating effective teaching and learning environments: First results from TALIS. Paris : OCDE. Disponible sur Internet à l'adresse : <http://www.oecd. org/document/54/0,374, en_2649 39263231_42980662_1_1_1_1,00.html> (consulté le 26 janvier 2011).

OTTESEN E. (2007). "Teachers "in the making": Building accounts of teaching ". Teaching and Teacher Education, vol. 23, n 5, p. 612-623.

PAQUAY L. (2005). "Devenir des enseignants et formateurs professionnels dans une organisation apprenante ? De l'utopie à la réalité ". European Journal of Teacher Education, vol. 28, $\mathrm{n}^{\circ}$ 2, p. 111-128.

PARKER-KATZ M. \& BAY M. (2008). "Conceptualizing mentor knowledge: Learning from the insiders ". Teaching and Teacher Education, vol. 24, n 5, p. 12591269.

PERRENOUD P., ALTET M., LESSARD C. \& PAQUAY L. (2008). Conflits de savoirs en formation des enseignants. Entre savoirs issus de la recherche et savoirs issus de l'expérience. Bruxelles: De Boeck.

PERRY N., HUTCHINSON L. \& THAUBERGER C. (2008). " Talking about teaching self-regulated learning: Scaffolding student teachers' development and use of practices that promote self-regulated learning ". International Journal of Educational Research, vol. 47, $n^{\circ} 2$, p. 97-108.

RAYMOND D. \& LENOIR Y. (1998). " Enseignants de métier et formation initiale : une problématique divergente et complexe ". In D. Raymond \& Y. Lenoir (dir.), Enseignants de métier et formation initiale. Bruxelles : De Boeck.

RICE E. (2002). "The collaboration process in Professional Development School: Results of a meta-ethnography, 1990-1998 ». Journal of Teacher Education, vol. 53, $n^{\circ} 1$, p. 55-67.

ROGOFF B., MATUSOV E. \& WHITE C. (1996). « Models of teaching and learning: Participation in a community of learners ". In Handbook of education and human development. Oxford: Blackwell, p. 389-414.

ROSS J. (1995). "Professional Development Schools: Prospects for institutionalization ». Teaching and Teacher Education, vol. 11, n 2, p. 195-201.

SIM C. (2006). "Preparing the professional experiences incorporating preservice teachers as communities of practice ". Teacher and Teaching Education, vol. 22, $\mathrm{n}^{\circ}$ 1, p. 77-83.

SMAGORINSKY P., COOK L., JACKSON A. \& FRY P. (2004). "Tensions in learning to teach: Accommodation and the development of a teaching identity ". Journal of Teacher Education, vol. 55, n 1, p. 8-24.

SMITH E. \& EVANS C. (2008). "Providing effective mentoring for alternate route beginning teachers ". The Teacher Educator, vol. $43, \mathrm{n}^{\circ}$ 4, p. 249-278.
SMITH K. (2008). "Améliorer la qualité de l'éducation par le biais de partenariats ". In CSEE, Rapport du séminaire IE/CSEE sur la formation des enseignants. Bruxelles : Comité syndical européen de l'éducation. Disponible sur Internet à l'adresse : <http://etuce.homestead.com/ CSEE_2010_publicationsbis.html> (consulté le 26 janvier 2011).

SMITH K. \& LEV-ARI L. (2005). " The place of the practicum in pre-service teacher education: The voice of the students ». Asia-Pacific Journal of Teacher Education, vol. 33, n 3, p. 289-302.

STANULIS R. \& FOLDEN R. (2009). " Intensive mentoring as a way to help beginning teachers develop balanced instruction ". Journal of Teacher Education, vol. 60, $n^{\circ} 2$, p. 112-122.

STOTSKY S. (2006). « Who should be accountable for what beginning teachers need to know? "Journal of Teacher Education, vol. 57, n 3, p. 256-268.

SUTHERLAND L., SCANLON L. \& SPERRING A. (2005). "New directions in preparing professionals: Examining issues in engaging students in communities of practice through a school-university partnership ». Teaching and Teacher Education, vol. 21, $\mathrm{n}^{\circ}$ 1, p. 79-92.

TEITEL L. (1998). " Separations, divorces, and open marriages in Professional Development School partnerships ". Journal of Teacher Education, vol. 49, $\mathrm{n}^{\circ} 2$, p. 85-96.

THIES-SPRINTHALL L. (1986). «A collaborative approach for mentor training: A working model ». Journal of Teacher Education, vol. 37, $\mathrm{n}^{\circ}$ 1, p. 13-20.

TILEMA H. (2009). " Assessment for learning to teach: Appraisal of practice teaching lessons by mentors, supervisors, and student teachers ". Journal of Teacher Education, vol. 60, $\mathrm{n}^{\circ}$ 2, p. 155-167.

TSUI A., LOPEZ-REAL F., LAW Y., TANG R. \& SHUM M. (2001). "Roles and relationships in tripartite supervisory conferencing processes ». Journal of Curriculum and Supervision, vol. $16, \mathrm{n}^{\circ} 4$, p. 322-344.

TWOMBLY S., WOLF-WENDEL L., WILLIAMS J. \& GREEN P. (2006). "Searching for the next generation of teacher educators ". Journal of Teacher Education, vol. $57, n^{\circ} 5$, p. 498-511.

VANHULLE S., MERHAN F. \& RONVEAU C. (2007). « Du principe d'alternance aux alternances en formation des adultes et des enseignants : un état de la question ". In F. Merhan, C. Ronveau \& S. Vanhulle (dir.), Alternances en formation. Bruxelles: De Boeck, p. 7-45.

VELZEN C. van \& VOLMAN M. (2009). "The activities of a school-based teacher educator: A theoretical and empirical exploration ". European Journal of Teacher Education, vol. $32, \mathrm{n}^{\circ} 4$, p. 345-367.

VELZEN C. van, BEZZINA C. \& LORIST P. (2009). "Partnerships between schools and teacher education institutes ». In A. Swennen \& M. van der Klink (dir.), Becoming a teacher educator. Dordrecht : Springer, p. 59-73.

VESCIO V., ROSS D. \& ADAMS A. (2008). " A review of research on the impact of professional learning communities on teaching practice and student learning ". Teaching and Teacher Education, vol. 24, $\mathrm{n}^{\circ} 1$, p. $80-91$ 
WANG J., STRONG M. \& ODELL S. (2004). « Mentor-novice conversations about teaching: A comparison of two US and two Chinese cases ". Teachers College Record, vol. $106, n^{\circ} 4$, p. $775-813$.

WATSON T. (2002). Organising and managing work. Harlow : Pearson.

WENGER E. (1998). Communities of practice. Cambridge : Cambridge University Press.

WENGER E. \& GERVAIS F. (2005). La théorie des communautés de pratique. Québec : Presses de l'Université Laval.

WENGER E., McDERMOTT R. \& SNYDER W. (2002). Cultivating communities of practice: $A$ quide to managing knowledge. Boston : Harvard Business School Press.

WHITEHEAD J. \& FITZGERALD B. (2007). " Experiencing and evidencing learning through self study: New ways of working with mentors and trainees in a training school partnership ". Teaching and Teacher Education, vol. $23, \mathrm{n}^{\circ} 1$, p. $1-12$
YENDOL-SILVA D. \& FICHTMAN DANA N. (2004). "Encountering new spaces: Teachers developing voice within a Professional Development School ». Journal of Teacher Education, vol. 55, n², p. 128-140.

ZEEGERS M. (2005). " From supervising practica to mentoring professional experience: Possibilities for education students ». Teaching Education, vol. 16, $\mathrm{n}^{\circ} 4$, p. 349-357.

ZEICHNER K. (1992). " Rethinking the practicum in the Professional Development School partnership ". Journal of Teacher Education, vol. 43, $n^{\circ}$ 4, p. 296-307.

ZEICHNER K. (2006). "Reflections of a university-based teacher educator on the future of college and university based teacher education ". Journal of Teacher Education, vol. 57, n 3 , p. 326-340.

ZEICHNER K. (2010). " Rethinking the connections between campus courses and field experiences in college- and university-based teacher education ". Journal of Teacher Education, vol. 61, n 1-2, p. 88-99. 